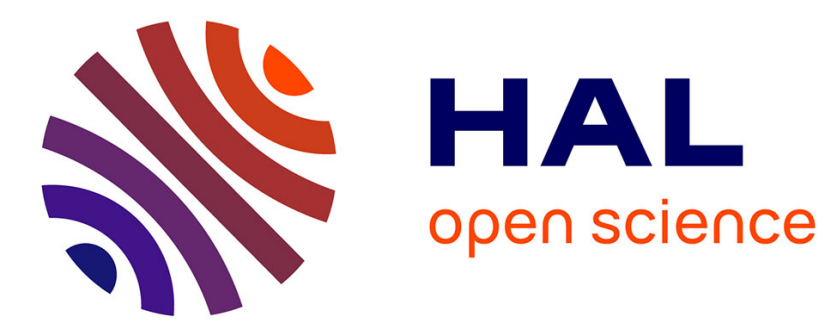

\title{
Multi-line Transmission for 3d Ultrasound Imaging: An Experimental Study
}

Emilia Badescu, Denis Bujoreanu, Lorena Petrusca, Denis Friboulet, Hervé Liebgott

\section{- To cite this version:}

Emilia Badescu, Denis Bujoreanu, Lorena Petrusca, Denis Friboulet, Hervé Liebgott. Multi-line Transmission for 3d Ultrasound Imaging: An Experimental Study. 2017 IEEE International Ultrasonic Symposium (IUS), Sep 2017, Washington, United States. hal-01599744

HAL Id: hal-01599744

https://hal.science/hal-01599744

Submitted on 25 Mar 2019

HAL is a multi-disciplinary open access archive for the deposit and dissemination of scientific research documents, whether they are published or not. The documents may come from teaching and research institutions in France or abroad, or from public or private research centers.
L'archive ouverte pluridisciplinaire HAL, est destinée au dépôt et à la diffusion de documents scientifiques de niveau recherche, publiés ou non, émanant des établissements d'enseignement et de recherche français ou étrangers, des laboratoires publics ou privés. 


\title{
MUlti-Line TranSMiSSION FOR 3D ULTRASOUND IMAGING: AN EXPERIMENTAL STUDY
}

\author{
Emilia Badescu, Denis Bujoreanu, Lorena Petrusca, Denis Friboulet, Hervé Liebgott \\ Univ Lyon, INSA-Lyon, Université Claude Bernard Lyon 1, UJM-Saint Etienne, CNRS, Inserm, CREATIS UMR 5220, U1206, \\ F-69100, LYON, France \\ Email: emilia.badescu@creatis.insa-lyon.fr
}

\begin{abstract}
Achieving a high frame rate in echocardiography is highly important for quantifying the short phases of the cardiac cycle that contain valuable information for medical diagnosis. Additionally, the 3D quantitative assessment of the heart would significantly improve the current measurements used in daily clinical routine. Nevertheless obtaining ultrafast images remains a challenge due to the trade-off between the image quality and a high frame rate, especially when volumetric data is acquired. Among the current ultrafast imaging methods, multi-linetransmit imaging (MLT) provides an increased frame rate but in the same time mostly preserves the image quality. In this paper we present the first real-time experimental implementation of the MLT in 3D ultrasound. The results indicate the potential of 3D MLT for achieving high contrast and resolution while increasing the frame rate. This study thus demonstrates the feasibility of 3D MLT in real-time and extends its possible applications to dynamic cardiac imaging.
\end{abstract}

Keywords-ultrasound, high frame rate, multi-line trasmit, $3 D$ imaging, experimental validation

\section{INTRODUCTION}

Ultrafast imaging is highly important in echocardiography for capturing rapid events that could be significant for diagnosis. Even if 3D ultrasound benefit from an increased clinical interest due to the possibility to assess accurate quantitative measurements, achieving a high frame rate remains a real challenge in 3D imaging [1].

Conventionally, with single focused transmissions, the frame rate can be increased by narrowing the field of view or diminishing the line density, which implies reducing the number of transmit events. In any case, the increase in frame rate comes with a compromise in terms of image quality (reducing the sector size or spatial resolution). As a solution to this inconvenient, multi-line acquisition (MLA) has been proposed and implemented on most of the clinical scanners [2]. This method is based on reconstructing multiple image lines in parallel from one emitted beam which results in a gain in frame rate related to the number of parallel acquisitions. However, MLA requires the broadening of the transmit beam which can be obtained by reducing the aperture size and therefore the transmitted energy. A lower transmit energy compromises the image quality by diminishing the signal to noise ratio (SNR).

Other approaches propose using electrocardiographic (ECG) retrospective gating for combining several subsectors over several cardiac cycles [3], [4]. But this technique may fail in patients presenting significant difference between cardiac cycles [1]. Despite the improvement in frame rate, these methods are still limited in current 3D implementation at frame rates on the order of a few tens of $\mathrm{Hz}$.

Several approaches have been proposed in literature to increase further the frame rate such as plane/diverging wave imaging [5], [6]. However, the high temporal resolution is achieved to the detriment of the spatial resolution. Even though spatial compounding allows coping with this limitation [7], the compounding reduces the frame rate.

Alternatively, several studies showed that multi-linetransmit imaging (MLT) provides an increased frame rate but in the same time mostly preserves the image quality. However, most of existing MLT analysis focused on 2D ultrasound imaging [8]-[11] or they were limited to $3 \mathrm{D}$ simulations [12]. Moreover, the latest implementation of this method in 3D, even if tested experimentally, is based on generating the MLT data synthetically by summing up the raw data before beamforming [13].

In this paper we present the first implementation of a realtime 3D MLT acquisition scheme on a research ultrasound system. Our aim is to prove its feasibility and to compare it with conventional focused imaging

\section{METHODS}

\section{A. Multi-line transmission}

The increase in frame rate is achieved by transmitting simultaneously several steered focused beams as described in 2D by Tong et al. [14]. In order to study the compromise between the high frame rate and image quality the number of MLT was varied from 4 to 8 and 16 . The transmission scheme of the three transmission set-ups is represented in Figure 1. As a general principle, the angular aperture is divided into 32 angles $\left(\alpha_{Y}, Y=1.32\right)$ between $\left[-30^{\circ}, 30^{\circ}\right]$ in elevational direction and 32 angles in azimuthal directional 
$\left(\theta_{X}, X=1 . .32\right)$. Considering $\mathrm{n}$ the number of simultaneous beams $(n=[4,8,16])$, for each set of $n T X=32 / n$ transmissions $\theta_{X}$ is kept fixed while $\alpha_{Y}$ is varied according to $\alpha_{i T X}, \alpha_{i T X+n T X}, \ldots ., \alpha_{i T X+(n-1) \cdot n T X}$, where $i T X=1 . . n T X$. Therefore, for each transmission the following pairs of angles are used to focalize simultaneously in $\mathrm{n}$ different directions: $\left(\theta_{j}, \alpha_{i T X}\right),\left(\theta_{j}, \alpha_{i T X+n T X}\right), \ldots,\left(\theta_{j}, \alpha_{i T X+(n-1) \cdot n T X}\right)$, where $\theta_{j}$ is changed for each set of $n T X$ transmissions in the same order as the angles in the elevational direction.
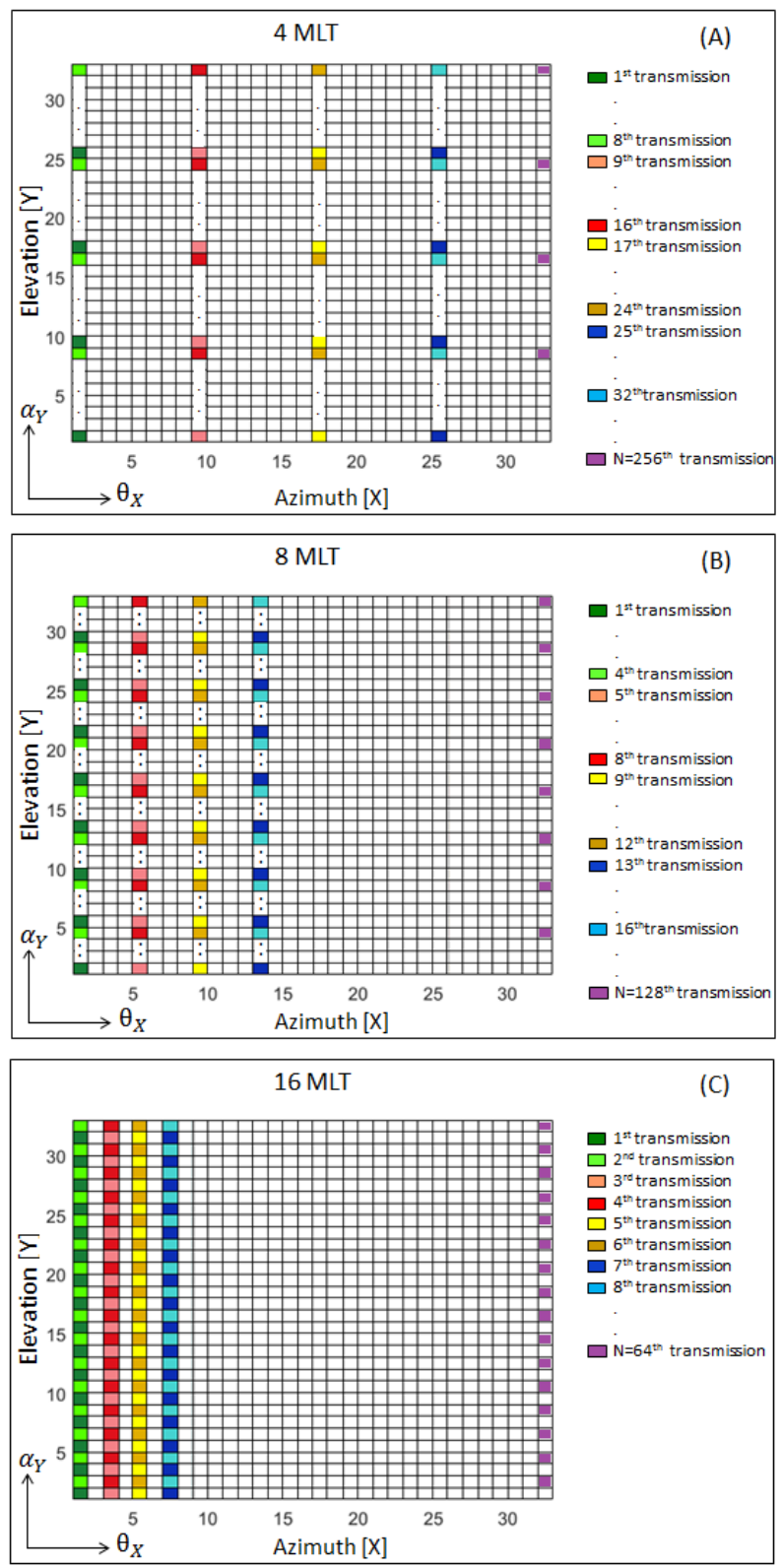

Figure 1: Transmission scheme for 4 MLT (A), 8 MLT (B) and 16 MLT (C)
In order to transmit multiple focused beams simultaneously, a superposition of waveforms needs to be applied to each element of the transducer. The Verasonics system allows the user to define arbitrary waveforms by using pulse codes [15]. Yet, as the number of multiple transmission increases, the number of superposed waveforms increases. Consequently, the pulse which codes the resulting waveform becomes more complex, requiring long transmits sequences that compromise the (thermal) safety of the imaged media and of the transducer. A solution for dealing with safety issues is to use short waveforms in transmission.

However, the consequence of using Dirac coded waveforms in transmission instead of longer waveforms such as a 3 cycle sinusoidal signal is illustrated in Figure 2, for a focused transmission.
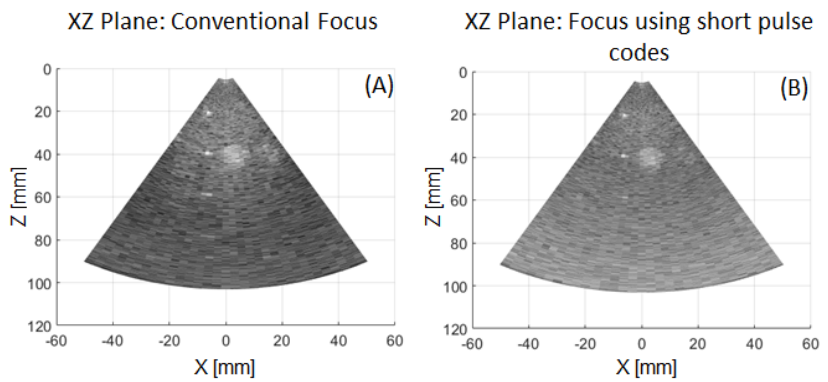

Figure 2: Focus sequence: $\mathrm{XZ}$ plane obtained by using 3 cycle signal (A), short coded pulse (B)

As it can be observed, using short pulse codes results in an improvement of axial resolution but with the tradeoff of lower contrast. The contrast to noise ratio (CNR) was calculated between the hyperechoic cyst placed at the focal point and the background, according to the expression:

$C N R=20 \log _{10}\left(\frac{\left|\mu_{b c k}-\mu_{c y s t}\right|}{\sqrt{\sigma_{b c k}^{2}+\sigma_{c y s t}^{2}}}\right)$

where $\mu_{b c k}, \mu_{c y s t}$ are mean and $\sigma_{b c k}^{2}, \sigma_{c y s t}^{2}$ are the corresponding variances of the background and the cyst regions calculated for a B-Mode image, having a dynamic range of $60 \mathrm{~dB}$.

The CNR decreased from $7.48 \mathrm{~dB}$ to $5.03 \mathrm{~dB}$ when short pulse codes were used. This is due to the low energy carried by the short excitation waveforms that results in a low Signal to Noise Ratio (SNR) for the received signals. The contrast degradation is more noticeable as the distance to the focus and the penetration depth are increased. For example, the media points placed at 80 and $90 \mathrm{~mm}$ are no longer visible for the image obtained using pulse codes.

Since our acquisition system allows the implementation of MLT just by using short pulse codes, for a fair analysis the 3D MLT acquisitions were compared in this study with a focused 
transmission obtained also using short excitation signals, as the one shown in Figure 2 (B).

\section{B. Experimental set-up}

Data acquisition was performed by using 4 Verasonics systems synchronized to drive a $32 \times 32$ elements Vermon probe having a central frequency of $2.97 \mathrm{MHz}$ [16]. The pitch was $0.3 \mathrm{~mm}$ and the images were acquired using a sampling frequency of $11.9 \mathrm{MHz}$. A volume of $32^{\circ} \times 32^{\circ}$ was insonified having a depth of $10.35 \mathrm{~cm}$. The focal point was set to $4 \mathrm{~cm}$.

\section{RESULTS}

The feasibility of 3D MLT was evaluated based on the data acquired on a CIRS ultrasound phantom (Model 054GS). Figure 3 illustrates three sections of its 3D volume, obtained by steering 4 focused beams simultaneously.
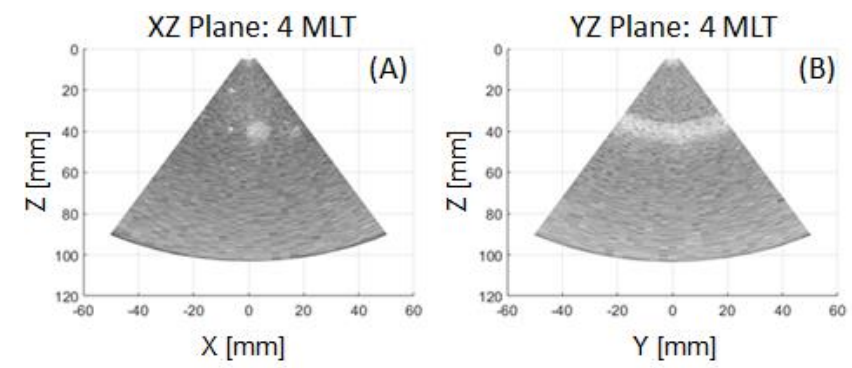

Surface at $Z=39 \mathrm{~mm}$

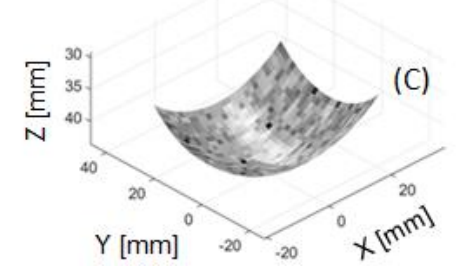

Figure 3: 3D results for 4 MLT.

(a) $\mathrm{XZ}$ and (b) YZ reconstructed planes and (c) a surface at a constant depth $(\mathrm{Z}=39 \mathrm{~mm})$

Rectangular (i.e. no) apodization was used on both transmitting and receiving elements. For comparison, the same apodization was used for the conventional focused sequence.

Emitting several beams in the same time leads to nonnegligible cross-talks artefacts, which become visible as the number of transmissions increases. Such an artefact is presented in Figure 4 (D) for the media point placed at $20 \mathrm{~mm}$ when 16 MLT was used.
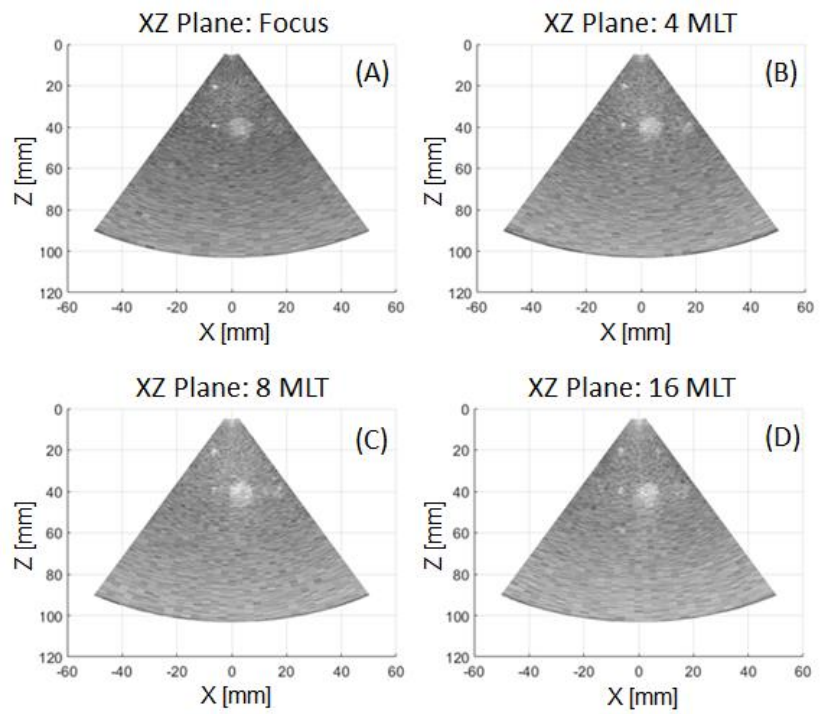

Figure 4: Comparison XZ planes for focus (A), 4 MLT (B), 8 MLT (C) and $16 \operatorname{MLT}(\mathrm{D})$

As shown in Figure 4, the image quality degrades with the number of simultaneous transmissions. For evaluating the degradation of the images, we calculated the CNR and the resolution which was computed using full width at half maximum (FWHM) at $-6 \mathrm{~dB}$. The contrast was investigated for a hyperechoic cylinder, whilst the resolution was measured for a media point, both placed on the focal point $(4 \mathrm{~cm})$.

While for 4 MLT the contrast has a value $(4.83 \mathrm{~dB})$ close to the one obtained using a conventional focused transmission $(5.03 \mathrm{~dB})$, it is reduced by $40 \%$ for 16 MLT. In the same time, the axial resolution degrades from $0.65 \mathrm{~mm}$ to $3.55 \mathrm{~mm}$ with a 16-fold increase in frame rate. All the contrast and axial resolution values are reported in Table I.

TABLE I: AXIAL RESOLUTION AND CONTRAST VALUES

\begin{tabular}{|l|l|l|l|l|}
\hline $\begin{array}{l}\text { Sequence/ } \\
\text { Image quality }\end{array}$ & Focus & $4 \mathrm{MLT}$ & $8 \mathrm{MLT}$ & $16 \mathrm{MLT}$ \\
\hline $\begin{array}{l}\text { Axial } \\
\text { Resolution }\end{array}$ & $0.65 \mathrm{~mm}$ & $1.25 \mathrm{~mm}$ & $2.1 \mathrm{~mm}$ & $3.55 \mathrm{~mm}$ \\
\hline Contrast & $5.03 \mathrm{~dB}$ & $4.83 \mathrm{~dB}$ & $4.05 \mathrm{~dB}$ & $3.61 \mathrm{~dB}$ \\
\hline
\end{tabular}

\section{DISCUSSION AND CONCLUSION}

The contrast and resolution values obtained when transmitting 4 focused beams simultaneously were comparable with those of a 3D focused sequence obtained by using the same emission parameters. The frame rate can be increased further but with the cost of the image quality. However, different methods that could improve the image 
quality and reduce the cross-talks even for a high frame-rate have been proposed in literature. Such techniques focused on aligning the transmit directions along the transverse diagonal of the transducer [12], using a proper apodization [13], using different frequency bands for the transmitted beams [17], [18] or the minimum variance beamformer [10], [19]. Most of these studies were conducted in 2D or in simulations in 3D. The exploration of these directions in experimental 3D will be the topic of future research.

The results presented in this paper indicate the experimental feasibility of MLT in 3D and its potential for achieving high contrast and resolution while increasing the frame acquisition rate, even for a simple transmission scheme and when no apodization is used. The real-time 3D MLT implementation presented in this study extends its possible applications to dynamic cardiac imaging.

\section{ACKNOWLEDGMENT}

This project has received funding from the European Union's Horizon 2020 research and innovation programme under the Marie Skłodowska-Curie grant agreement No 642612, VPH-CaSE (www.vph-case.eu). This work was performed within the framework of the LABEX PRIMES (ANR-11-LABX-0063) of Université de Lyon, within the program "Investissements d'Avenir" (ANR-11-IDEX-0007) operated by the French National Research Agency (ANR).

The authors would like to thank LabTAU for their contribution in the development of the $32 \times 32$ probe prototype compatible with a driving by 1 to 4 Verasonics Vantage 256 as well as for the provision of the probe and two Vantage 256 systems. Two Verasonics systems were acquired thanks to the program FEDER Saint-Etienne and Loire General Council in the framework of the project SonoCardioProtection supervised by Pierre Croisille.

\section{REFERENCES}

[1] M. Cikes, L. Tong, G. R. Sutherland, and J. D'hooge, "Ultrafast Cardiac Ultrasound Imaging," JACC Cardiovasc. Imaging, vol. 7, no. 8, pp. 812-823, Aug. 2014.

[2] D. P. Shattuck, M. D. Weinshenker, S. W. Smith, and O. T. von Ramm, "Explososcan: A parallel processing technique for high speed ultrasound imaging with linear phased arrays," J. Acoust. Soc. Am., vol. 75, no. 4, pp. 1273-1282, Apr. 1984.

[3] S. Shougang Wang, W.-N. Wei-ning Lee, J. Provost, J. Jianwen Luo, and E. E. Konofagou, "A composite high-frame-rate system for clinical cardiovascular imaging," IEEE Trans. Ultrason. Ferroelectr. Freq. Control, vol. 55, no. 10, pp. 2221-2233, Oct. 2008.
Konofagou, "ECG-gated, Mechanical and Electromechanical Wave Imaging of Cardiovascular Tissues In Vivo," Ultrasound Med. Biol., vol. 33, no. 7, pp. 1075-1085, Jul. 2007.

L. Sandrin, S. Catheline, M. Tanter, X. Hennequin, and M. Fink, "Time-Resolved Pulsed Elastography with Ultrafast Ultrasonic Imaging," Ultrason. Imaging, vol. 21, no. 4, pp. 259-272, Oct. 1999.

[6] H. Hasegawa and H. Kanai, "High-frame-rate echocardiography using diverging transmit beams and parallel receive beamforming," J. Med. Ultrason., vol. 38, no. 3, pp. 129-140, Jul. 2011.

[7] G. Montaldo, M. Tanter, J. Bercoff, N. Benech, and M. Fink, "Coherent plane-wave compounding for very high frame rate ultrasonography and transient elastography.," IEEE Trans. Ultrason. Ferroelectr. Freq. Control, vol. 56, no. 3, pp. 489-506, Mar. 2009.

[8] R. Mallart and M. Fink, "Improved imaging rate through simultaneous transmission of several ultrasound beams," 1992, pp. $120-130$.

[9] L. Tong, A. Ramalli, R. Jasaityte, P. Tortoli, and J. D'hooge, "Multi-Transmit Beam Forming for Fast Cardiac Imaging Experimental Validation and In Vivo Application," IEEE Trans. Med. Imaging, vol. 33, no. 6, pp. 1205-1219, Jun. 2014.

[10] A. Rabinovich, A. Feuer, and Z. Friedman, "Multi-line transmission combined with minimum variance beamforming in medical ultrasound imaging," IEEE Trans. Ultrason. Ferroelectr. Freq. Control, vol. 62, no. 5, pp. 814-827, May 2015.

[11] L. Demi, J. Viti, L. Kusters, F. Guidi, P. Tortoli, and M. Mischi, "Implementation of parallel transmit beamforming using orthogonal frequency division multiplexing-achievable resolution and interbeam interference," IEEE Trans. Ultrason. Ferroelectr. Freq. Control, vol. 60, no. 11, pp. 2310-2320, Nov. 2013.

[12] B. Denarie, T. Bjastad, and H. Torp, "Multi-line transmission in 3D with reduced crosstalk artifacts: a proof of concept study," IEEE Trans. Ultrason. Ferroelectr. Freq. Control, vol. 60, no. 8, pp. 1708-1718, Aug. 2013.

[13] A. Ortega, J. Provost, L. Tong, P. Santos, B. Heyde, M. Pernot, and J. D'hooge, "A Comparison of the Performance of Different Multiline Transmit Setups for Fast Volumetric Cardiac Ultrasound," IEEE Trans. Ultrason. Ferroelectr. Freq. Control, vol. 63, no. 12, pp. 2082-2091, Dec. 2016.

[14] L. Tong, H. Gao, and J. D'hooge, "Multi-transmit beam forming for fast cardiac imaging-a simulation study," IEEE Trans. Ultrason. Ferroelectr. Freq. Control, vol. 60, no. 8, pp. 1719-1731, Aug. 2013.

[15] J. A. Flynn, P. Kaczkowski, K. Linkhart, and R. E. Daigle, "Arbitrary waveforms using a tri-state transmit pulser," in 2013 IEEE International Ultrasonics Symposium (IUS), 2013, pp. 41-44.

[16] M. . Petrusca, L.; Varray, F.; Souchon, R.; Bernard, A.; Chapelon, J.Y.; Liebgott, H. ; N'Djin, W.A.; Viallon, "A new high channels density ultrasound platform for advanced 4D cardiac imaging," in IEEE Internation Ultrasonics Symposium, 2017.

[17] L. Demi, M. D. Verweij, and K. W. A. Van Dongen, "Parallel transmit beamforming using orthogonal frequency division multiplexing applied to harmonic Imaging-A feasibility study," IEEE Trans. Ultrason. Ferroelectr. Freq. Control, vol. 59, no. 11, pp. 2439-47, Nov. 2012.

[18] D. T. . Dubberstein and O. T. . von Ramm, "Methods and systems for ultrasound scanning using spatially and spectrally separated transmit ultrasound beams," US Patent 6159153 A, 2000.

[19] J. A. Mann and W. F. Walker, "A constrained adaptive beamformer for medical ultrasound: initial results," in 2002 IEEE Ultrasonics Symposium, 2002. Proceedings., vol. 2, pp. 1807-1810. 